\title{
Values of linear recurring sequences of vectors over finite fields
}

\author{
by \\ Gary L. Mullen (University Park, Penn.) and \\ IGOR ShPARLINSKI (Sydney, N.S.W.)
}

Consider the finite field $\mathbb{F}_{q}$ of $q=p^{r}$ elements where $p$ is a prime, $r \geq 1$. For an $n$-tuple $\left(v_{1}, \ldots, v_{n}\right)$ of vectors

$$
v_{i}=\left(v_{i, 1}, \ldots, v_{i, n}\right)^{T}
$$

from the $n$-dimensional vector space $\mathbb{F}_{q}^{n}$ over $\mathbb{F}_{q}$ and a polynomial

$$
f(x)=x^{n}-\sum_{i=0}^{n-1} a_{i} x^{i} \in \mathbb{F}_{q}[x],
$$

we consider the linear recurring sequence $S=\{s(k)\}$ defined by

$$
s(k)= \begin{cases}v_{k} & \text { if } k \leq n, \\ \sum_{i=0}^{n-1} a_{i} s(k-n+i) & \text { if } k>n .\end{cases}
$$

We note that the elements of the sequence $S$ can be considered as elements of the field $\mathbb{F}_{q^{n}}$ which is an $n$-dimensional vector space over $\mathbb{F}_{q}$.

It is easy to see that without loss of generality we can suppose that $f(0) \neq 0$. Thus, it is possible to define the order of $f$, denoted by $\tau$, as the least positive integer $t$ for which $f(x)$ divides $x^{t}-1$. It is known from [4] that the period of the sequence $S$ does not exceed $\tau$. For other details concerning polynomials and linear recurring sequence over $\mathbb{F}_{q}$, see [4].

In this paper we improve and generalize some results from the papers $[1],[2],[6],[7]$ which are also devoted to studying the distribution of values of linear recurring sequences over finite fields. For some applications of such sequences see [1], [2].

It is easy to check that if $\alpha, \mu \in \mathbb{F}_{q^{n}}$ then for any fixed basis of $\mathbb{F}_{q^{n}}$ over $\mathbb{F}_{q}$, the coordinate-vectors $\left\{c_{k}\right\}$ of the powers $\alpha \mu^{k}, k=1,2, \ldots$, satisfy such

The first author would like to thank the National Security Agency for partial support under grant agreement \#MDA904-92-H-3044. 
an equation corresponding to the minimal polynomial of $\alpha$ over $\mathbb{F}_{q}$. More generally, for an $n \times n$ matrix $A$ and a vector a over $\mathbb{F}_{q}$ the sequence $\left\{\mathbf{a} A^{k}\right\}$ satisfies such an equation corresponding to the minimal polynomial of $A$ over $\mathbb{F}_{q}$.

Thus, results on the distribution of values of such linear recurring sequences are related to the well-known discrete logarithm problem and to the orbit problem in finite fields (see [3] and [5] for background and references).

For an integer $P>1$ denote by $V(P)$ the set of all possible values which occur among the first $P$ elements $s_{1}, \ldots, s_{P}$ of the sequence $S$, and by $V$ the set of all possible vectors which occur among elements of the sequence $S$. In [1], some sufficient conditions were stated under which such a sequence consists of all nonzero elements of $\mathbb{F}_{q}^{n}$, i.e. when $V=\mathbb{F}_{q}^{n} \backslash\{(0, \ldots, 0)\}$ (or $V=\mathbb{F}_{q^{n}}^{*}$ if we consider $S$ as a sequence of elements of $\left.\mathbb{F}_{q^{n}}\right)$.

Denote by $m$ the dimension of the vector space generated by the initial vectors $\left(v_{1}, \ldots, v_{n}\right)$. It has been shown in [1] that in this case

$$
|V| \leq \min \left\{q^{m}, \tau\right\},
$$

where $|V|$ denotes the cardinality of the set $V$.

It is clear that the $n \times n$ matrix $\left(v_{1}, \ldots, v_{n}\right)$ contains $m \leq n$ linearly independent rows which we denote by $w_{i}=\left(w_{i, 1}, \ldots, w_{i, n}\right), i=1, \ldots, m$. Thus we can define $m$ linear recurring sequences $W_{i}=\left\{w_{i}(k)\right\}$ by

$$
w_{i}(k)= \begin{cases}w_{i, k} & \text { if } k \leq n, \\ \sum_{j=0}^{n-1} a_{j} w_{i}(k-n+j) & \text { if } k>n\end{cases}
$$

(with the same characteristic polynomial $f(x)$ ) which are linearly independent over $\mathbb{F}_{q}$.

Note that the sequence of vectors $\left(w_{1}(k), \ldots, w_{m}(k)\right)^{T}, k=1,2, \ldots$, is periodic with the minimal period $T$ dividing $\tau$. Moreover, if $f(x)$ is an irreducible polynomial then $T=\tau$.

Denote by $M_{m}(P)$ the number of different vectors which occur among

$$
\left(w_{1}(k), \ldots, w_{m}(k)\right)^{T}, \quad k=1, \ldots, P,
$$

so that $|V(P)|=M_{m}(P)$.

Let us set for brevity

$$
M_{m}=M_{m}(\tau), \quad M(P)=M_{1}(P), \quad M=M(\tau) .
$$

Then it has been shown in Theorem 3.2 of [1] that if $f(x)$ is a primitive polynomial of degree $n$ (i.e. $\tau=q^{n}-1$ ) and $m<n$ then $M_{m}=q^{m}$. Furthermore, when $m=1$ it has been noted in [1] that there is an asymptotic formula for the number of solutions of certain equations with a linear recurring sequence which enables the authors to prove that $M=q$ whenever $\tau>(q-1) q^{n / 2}$.

Now we are going to show that the result of [6] for systems of equations with linear recurring sequences allows us to extend this result to any $m \geq 1$. 
Set $t=\tau / \operatorname{gcd}(\tau, q-1)$.

THEOREM 1. Let the dimension of the vector space generated by the initial vectors $\left(v_{1}, \ldots, v_{n}\right)$ be $m$ and let $f(x)$ be an irreducible polynomial over $\mathbb{F}_{q}$ of order $\tau$. Then there exists an absolute constant $C>0$ such that if $P>C q^{m+(n-1) / 2} \log \tau$ then $M_{m}(P)=M_{m}$.

Proof. For $\theta_{1}, \ldots, \theta_{m} \in \mathbb{F}_{q}$ denote by $N_{P}\left(\theta_{1}, \ldots, \theta_{m}\right)$ the number of solutions of the system of equations

$$
w_{i}(k)=\theta_{i}, \quad i=1, \ldots, m, 1 \leq k \leq P .
$$

It follows from [6] that

$$
N_{P}(0, \ldots, 0)=P / q^{m}+O\left(q^{n / 2-1} \log \tau\right)
$$

for $P \leq t$ and that

$$
N_{P}\left(\theta_{1}, \ldots, \theta_{m}\right)=P / q^{m}+O\left(q^{(n-1) / 2} \log \tau\right)
$$

for $P \leq \tau$, for any non-zero tuple $\left(\theta_{1}, \ldots, \theta_{m}\right)$, with absolute implied constants in the $O$-symbol (see Theorems 1 and 2 of [6], respectively). From (2) and (3) we get that

$$
N_{P}\left(\theta_{1}, \ldots, \theta_{m}\right)=\frac{P}{\tau} N_{\tau}\left(\theta_{1}, \ldots, \theta_{m}\right)+O\left(q^{(n-1) / 2} \log \tau\right)
$$

for any $P$ and any tuple $\left(\theta_{1}, \ldots, \theta_{m}\right)$.

It has been noted in [6] that in the cases $P=t$ and $P=\tau$ the logarithmic factor in the error terms of (2) and (3) respectively can be omitted. Thus, there is some absolute constant $C>0$ such that if $t>C q^{m+n / 2-1}$ and $\tau>C q^{m+(n-1) / 2}$ then $M_{m}=q^{m}$. This result together with Theorem 1 allows us to easily formulate conditions under which $M_{m}(P)=q^{m}$. If we do not consider the zero tuple $(0, \ldots, 0)$, then similarly we get $M_{m}(P) \geq q^{m}-1$ and $M_{m} \geq q^{m}-1$ for $\tau \geq P>C n q^{m+(n-1) / 2} \log q$ and $\tau>C n q^{m+(n-1) / 2}$, respectively.

Moreover, it is an easy matter to explicitly compute constants in all of the above mentioned bounds (in fact, they are quite reasonable, about 1).

Since $t \geq \tau / q$ the condition $\tau>C n q^{m+n / 2}$ guarantees that $M_{m}=q^{m}$. This is a generalization (up to the constant $C$ ) of the above mentioned result concerning the case $m=1$. An evident deficiency of Theorem 1 is that it can be utilized only if the period is sufficiently large.

The following results give other lower bounds for $M_{m}(P)$ that are nontrivial for any $\tau$. First we get new lower bounds for the number $M(P)$. It is easy to prove that $M>\tau^{1 / n}$ and $M(P)>(P-n)^{1 / n}$ (see the proof of Theorem 3 below). We show that for fields $\mathbb{F}_{q}$ of small characteristic $p$ this bound can be improved. 
We need the following refinement of Theorem 1 of [7]:

$$
M(P) \geq \min \left\{M, P\left(\begin{array}{c}
n+p-2 \\
p-1
\end{array}\right)^{-l}\right\}
$$

where $l$ is the least integer with $M(P) \leq p^{l}$.

In order to obtain this result we can replace the trivial bound $M(P) \leq$ $q=p^{r}$ in the proof of Theorem 1 of [7] with the inequality $M(P) \leq p^{l}$.

TheOREM 2. We have the bound

$$
M(P) \geq \min \left\{M, p^{-1} P^{\log p /(\log p+p \log n)}\right\} .
$$

Proof. It follows from (4) that $M(P)<M$ implies

$$
\begin{aligned}
P & \leq M(P)\left(\begin{array}{c}
n+p-2 \\
p-1
\end{array}\right)^{l} \leq M(P) n^{p l}=M(P) p^{l p \log n / \log p} \\
& <(p M(P))^{p \log n / \log p+1}
\end{aligned}
$$

since, by definition, $p^{l-1}<M(P)$.

The next theorem generalizes the above result to the $m$-dimensional case.

THEOREM 3. Let $f(x)$ be an irreducible polynomial over $\mathbb{F}_{q}$ of order $\tau$. Then we have the bounds

$$
M_{m}(P) \geq(P-n+m)^{1 /(n-m+1)}
$$

and

$$
M_{m}(P) \geq \min \left\{\tau^{1 /(n-m+1)}, p^{-1} P^{\log p /(\log p+p \log (n-m+1))}\right\} .
$$

Pr o of. Let $\lambda_{1}, \ldots, \lambda_{n}$ be the roots of $f(x)$ (lying in $\mathbb{F}_{q^{n}}$ ). Then we have the representations

$$
w_{i}(k)=\sum_{j=1}^{n} \alpha_{i, j} \lambda_{j}^{k}, \quad i=1, \ldots, m, k=1,2, \ldots,
$$

for some $\alpha_{i, j} \in \mathbb{F}_{q^{n}}, i=1, \ldots, m, j=1, \ldots, n$.

Let $\beta_{1}, \ldots, \beta_{m} \in \mathbb{F}_{q^{n}}$ be any nonzero solution of the following system of $m-1$ linear homogeneous equations

$$
\sum_{i=1}^{m} \alpha_{i, j} \beta_{i}=0, \quad j=n-m+2, \ldots, n .
$$

Define the sequence

$$
\omega(k)=\sum_{i=1}^{m} \beta_{i} w_{i}(k)
$$


Then for some $\gamma_{j}, j=1, \ldots, n-m+1$, we have

$$
\omega(k)=\sum_{j=1}^{n-m+1} \gamma_{j} \lambda_{j}^{k}, \quad k=1,2, \ldots
$$

It is evident that the sequences $W_{i}, i=1, \ldots, m$ are linearly independent over $\mathbb{F}_{q^{n}}$ as well. Thus $\Omega=\{\omega(k)\}$ is a nonzero linear recurring sequence of elements of the field $\mathbb{F}_{q^{n}}$ of order at most $n-m+1$. Now we are going to show that the period of the sequence $\Omega$ equals $\tau$.

Since $f(x)$ is irreducible, the condition $f(x) \mid\left(x^{\tau}-1\right)$ is equivalent to $\lambda_{j}^{\tau}-1=0, j=1, \ldots, n$, and moreover all of these equalities are equivalent. Therefore, if $\omega(k+T)=\omega(k), k=1,2, \ldots$, and $\lambda_{j}^{T}-1 \neq 0, j=1, \ldots, n$ then we see that the system

$$
\sum_{j=1}^{n-m+1} \psi_{j} \lambda_{j}^{k}=0, \quad k=1, \ldots, n-m+1,
$$

has a nonzero solution $\psi_{j}=\gamma_{j}\left(\lambda_{j}^{T}-1\right), j=1, \ldots, n-m+1$, which is impossible.

Evidently, $M_{m}(P)$ is greater than or equal to the number of different values which occur among $\omega(1), \ldots, \omega(P)$. On the other hand, taking into account that $\Omega$ is a linear recurring sequence of order $n-m+1$ and of period $\tau$, we conclude that for $P \leq \tau$ all tuples

$$
(\omega(k), \ldots, \omega(k+n-m)), \quad k=1, \ldots, P-n+m,
$$

are pairwise different. Thus $M_{m}(P)^{n-m+1} \geq P$ and we obtain the first bound.

It is easy to note that for $P=\tau$ we could consider $\tau$ pairwise different tuples

$$
(\omega(k), \ldots, \omega(k+n-m)), \quad k=1, \ldots, \tau,
$$

rather than $\tau-n+m$. Then the sequence $\Omega$ takes at least $\tau^{1 /(n-m+1)}$ different values. Hence

$$
M_{m} \geq \tau^{1 /(n-m+1)},
$$

and applying Theorem 2 we get the second bound.

The following theorem is a generalization and an improvement of Theorem 1 of [7]. It is nontrivial for all $q$ but is especially effective when $p$ is a fixed prime.

Theorem 4. For $P>\left(p M_{m}\right)^{p \log n / \log p+1}$ we have $M_{m}(P)=M_{m}$.

Proof. Let $\theta_{1}, \ldots, \theta_{m}$ be a basis of the field $\mathbb{F}_{q^{m}}$ over $\mathbb{F}_{q}$. Applying Theorem 2 to the linear recurring sequence

$$
u(k)=\theta_{1} w_{1}(k)+\ldots+\theta_{m} w_{m}(k), \quad k=1,2, \ldots,
$$


over $\mathbb{F}_{q^{m}}$ we get

$$
M_{m}(P) \geq \min \left\{M_{m}, p^{-1} P^{\log p /(\log p+p \log n)}\right\},
$$

and the result follows.

Since $M_{m} \leq q^{m}$ the statement of the theorem holds for

$$
P>(p q)^{m(p \log n / \log p+1)} .
$$

Thus, for $p$ fixed, $M_{m}(P)=M_{m}$ for some $P=\exp (O(m \log q \log n))$. In particular, if $m$ and $q$ are fixed, then the number of vectors $\left(w_{1}(k), \ldots\right.$, $\left.w_{m}(k)\right)$ that we need to compute in order to determine the set of all possible distinct values, is bounded by $n^{O(1)}$, i.e. the computation can be done in polynomial time.

In fact, when $q$ is fixed, for an arbitrary $m$ the number of vectors which we need to compute can be estimated by $\exp \left(O\left(\log M_{m} \log n\right)\right)$ which is a quasi-polynomial function $\exp \left(\log ^{2} L\right)$ in the total size $L=L_{i}+L_{o}$ of the input $L_{i}=O(n)$ and of the output $L_{o}=O\left(m M_{m}\right)$. However, we do not know any upper bounds for $M_{m}$ (excepting $M_{m} \leq q^{m}$ ).

Acknowledgement. We would like to thank the referee for several helpful comments.

\section{References}

[1] W.-S. Chou and G. L. Mullen, Generating linear spans over finite fields, Acta Arith. 61 (1992), 183-191.

[2] R. Fitzgerald and J. Yucas, On generating linear spans over $\mathrm{GF}(p)$, Congr. Numer. 69 (1989), 55-60.

[3] R. Kannan and R. J. Lipton, Polynomial-time algorithm for the orbit problem, J. Assoc. Comput. Mach. 33 (1986), 808-821.

[4] R. Lidl and H. Niederreiter, Finite Fields, Encyclopedia Math. Appl. 20, Addison-Wesley, Reading, Mass., 1983 (now distributed by Cambridge Univ. Press).

[5] K. S. McCurley, The discrete logarithm problem, in: Cryptology and Computational Number Theory, C. Pomerance (ed.), Proc. Sympos. Appl. Math. 42, Amer. Math. Soc., 1990, 49-74.

[6] I. Shparlinski, On the distribution of recurring sequences, Problemy Peredachi Informatsii 25 (2) (1989), 46-53 (in Russian).

[7] - On the distribution of values of recurring sequences and the Bell numbers in finite fields, European J. Combin. 12 (1991), 81-87.

MATHEMATICS DEPARTMENT THE PENNSYLVANIA STATE UNIVERSITY UNIVERSITY PARK, PENNSYLVANIA 16802 U.S.A.

E-mail: MULLEN@MATH.PSU.EDU
SCHOOL OF MPCE MACQUARIE UNIVERSITY SYDNEY, NEW SOUTH WALES 2109 AUSTRALIA E-mail: IGOR@MACADAM.MPCE.MQ.EDU.AU

Received on 10.9.1992

and in revised form on 15.4.1993 